\title{
ApoE4 Decreases Spine Density and Dendritic Complexity in Cortical Neurons In Vivo
}

\author{
Sonya B. Dumanis, ${ }^{1 *}$ Joseph A. Tesoriero, ${ }^{1 \star}$ Lenard W. Babus, ${ }^{1}$ Madeline T. Nguyen, ${ }^{3}$ Justin H. Trotter, ${ }^{4}$ Mary Jo Ladu, ${ }^{5}$ \\ Edwin J. Weeber, ${ }^{4}$ R. Scott Turner, ${ }^{2}$ Baoji Xu, ${ }^{3}$ G. William Rebeck, ${ }^{1}$ and Hyang-Sook Hoe ${ }^{1,2}$ \\ Departments of ${ }^{1}$ Neuroscience, ${ }^{2}$ Neurology, and ${ }^{3}$ Pharmacology, Georgetown University Medical Center, Washington, DC 20057, ${ }^{4}$ Department of Molecular \\ Pharmacology and Physiology, Jonnie B. Byrd, Sr. Alzheimer's Center \& Research Institute, University of South Florida, Tampa, Florida 33612, and \\ ${ }^{5}$ Department of Anatomy and Cell Biology, University of Illinois at Chicago, Chicago, Illinois 60612
}

The three human alleles of apolipoprotein E (APOE) differentially influence outcome after CNS injury and affect one's risk of developing Alzheimer's disease (AD). It remains unclear how ApoE isoforms contribute to various AD-related pathological changes (e.g., amyloid plaques and synaptic and neuron loss). Here, we systematically examined whether apoE isoforms (E2, E3, E4) exhibit differential effects on dendritic spine density and morphology in APOE targeted replacement (TR) mice, which lack AD pathological changes. Using Golgi staining, we found age-dependent effects of APOE4 on spine density in the cortex. The APOE4 TR mice had significantly reduced spine density at three independent time points ( 4 weeks, 3 months, and 1 year, $27.7 \% \pm 7.4 \%, 24.4 \% \pm 8.6 \%$, and $55.6 \% \pm 10.5 \%$, respectively) compared with APOE3 TR mice and APOE2 TR mice. Additionally, in APOE4 TR mice, shorter spines were evident compared with other APOE TR mice at 1 year. APOE2 TR mice exhibited longer spines as well as significantly increased apical dendritic arborization in the cortex compared with APOE4 and APOE3 TR mice at 4 weeks. However, there were no differences in spine density across APOE genotypes in hippocampus. These findings demonstrate that apoE isoforms differentially affect dendritic complexity and spine formation, suggesting a role for APOE genotypes not only in acute and chronic brain injuries including AD, but also in normal brain functions.

\section{Introduction}

Alzheimer's disease (AD) is characterized by extracellular amyloid $\beta$ plaques, intracellular hyperphosphorylated tau tangles, inflammation, gliosis, and brain atrophy resulting from synaptic and neuronal loss (DeKosky and Scheff, 1990). The strongest genetic risk factor for sporadic AD is apolipoprotein E (APOE) whose protein product is responsible for transport of lipids throughout the circulatory system (Herz and Beffert, 2000). APOE has three common alleles: $\varepsilon 2, \varepsilon 3$, and $\varepsilon 4$, which differ from each other by single amino acid substitutions at residues 112 and 158. The $\varepsilon 4$ allele increases the risk for developing $\mathrm{AD}$ (Rebeck et al., 1993; Strittmatter et al., 1993; Yoshizawa et al., 1994), while the $\varepsilon 2$ allele decreases this risk (Poirier et al., 1993). Interestingly, $\varepsilon 4$ is associated not only with $\mathrm{AD}$, but also with increased cognitive decline in elderly $\varepsilon 4$ carriers asymptomatic of AD (Plassman et al., 1997) and altered brain metabolism in young cognitively normal adults (Scarmeas et al., 2005; Filippini et al., 2009). Furthermore, $\varepsilon 4$ is linked to worse outcomes from

Received Aug. 17, 2009; revised 0ct. 9, 2009; accepted 0ct. 13, 2009.

This work was supported by National Institutes of Health Grants AG032330 (H.S.H.), AG032330-02S1 (H.S.H.), AG014473 (G.W.R.), and AG026478 (R.S.T.). J.A.T. and L.W.B. were supported by a grant to Georgetown University from the Undergraduate Program at the Howard Hughes Medical Institute. We thank Dr. Patrick Sullivan for generously providing us with the apoE targeted replacement mice. We also thank Drs. Dan Pak and Kea Joo Lee for expert technical assistance.

*S.B.D. and J.A.T. contributed equally to this work.

Correspondence should be addressed to Dr. Hyang-Sook Hoe, Departments of Neurology and Neuroscience, Georgetown University, 3970 Reservoir Road NW, Washington, DC 20057-1464. E-mail: hh69@georgetown.edu. DOI:10.1523/JNEUROSCI.4026-09.2009

Copyright $\odot 2009$ Society for Neuroscience ～0270-6474/09/2915317-06\$15.00/0
CNS injuries including traumatic brain injury (Teasdale et al., 1997), and may increase risk of cognitive decline in subjects with non-AD dementias (Daniele et al., 2009).

The APOE genotype may have a profound influence on the extent of disease-related synaptic deterioration due to its effects on dendritic growth. ApoE3 promotes, while apoE4 reduces, neuritic outgrowth in vitro (Nathan et al., 1994; Bellosta et al., 1995; Teter et al., 1999). In vivo and in vitro studies have also exhibited a role for apoE4 in reducing neurite sprouting (Buttini et al., 1999; Wang et al., 2005). Together, these findings led us to hypothesize that apoE4 may impede dendritic outgrowth and decrease the formation of new synapses, thereby contributing to disease development.

To test this hypothesis, we systematically examined the effect of all three apoE isoforms on spine formation in cortical layers II/III and in the dentate gyrus. Using APOE targeted replacement (TR) mice, we found that at all ages, APOE4 TR mice had decreased spine density in cortical neurons compared with APOE2 and APOE3 TR mice but not in dentate gyrus granule cells. Furthermore, APOE2 TR mice had increased dendritic complexity compared with APOE3 and APOE4 TR mice. These results show that APOE isoforms differentially affect neurite and dendritic spine morphologies in vivo, suggesting these effects could modulate normal brain functions as well as reparative functions affecting risk, prognosis, and disease course in many CNS disorders.

\section{Materials and Methods}

Mice. Human APOE2, APOE3, and APOE4 TR mice, which express each of the human APOE isoforms regulated by the endogenous murine 
APOE promoter (Wang et al., 2005) were donated by Dr. Patrick Sullivan (Duke University). Briefly, all three lines of APOE TR mice contain chimeric genes consisting of mouse $5^{\prime}$ regulatory sequences continuous with the noncoding region (mouse exon 1 ) followed by human exons (and introns) 2-4 (Sullivan et al., 1997). Mice were backcrossed to C57BL/6J mice eight times and therefore are $>99.6 \%$ C57BL/6J. APOE-deficient mice and C57BL/6J wild-type mice were obtained from The Jackson Laboratory.

Golgi staining and analysis of dendritic morphology in vivo. Golgi staining was performed on APOE2, APOE3, and APOE4 TR mice (4 weeks, 3 months, and 1 year; $n=4$ per genotype for each time point), APOE knock-out mice ( 4 weeks and 3 months; $n=4$ mice per genotype for each time point), and wild-type C57BL/6J mice ( 1 year; $n=4$ mice per genotype) For these experiments, we used the FD Rapid Golgi Stain kit (FD NeuroTechnologies) as previously described (Hoe et al., 2009). Briefly, freshly dissected brains were immersed in solution A and B for 2 weeks at room temperature and transferred into solution $\mathrm{C}$ for $24 \mathrm{~h}$ at $4^{\circ} \mathrm{C}$. The brains were sliced using a Vibratome (VT1000S; Leica) at a thickness of 150 $\mu \mathrm{m}$. Bright-field microscopy (Axioplan 2; Zeiss) images (at $63 \times$ magnification) were taken of pyramidal neurons in cortical layers II/III (56 neurons per genotype per time point), granule cells in the dentate gyrus ( $n=$ 28 neurons per genotype per time point), and CA1 pyramidal neurons $(n=56$ neurons per genotype). Images were coded, and dendritic spines counted in a blinded manner using Neurolucida software (MicroBrightField). All the spines counted were also measured for spine length as previously described (Pak et al., 2001).

Hippocampal culture. Primary hippocampal neurons from embryonic day 18 (E18)-E19 Sprague Dawley rats were cultured at $150 \mathrm{cells} / \mathrm{mm}^{2}$ as described previously (Pak et al., 2001). Primary hippocampal neurons (day in vitro 18) were transfected with GFP and treated with 500 nM recombinant apoE3 or apoE4 (Oxford Biomedical Research) or PBS as control for $48 \mathrm{~h}$. A total of 15 neurons from each group were analyzed.

Scholl analysis. 18 neurons in somatosensory cortical layers II/III were used for each group (APOE2, APOE3, and APOE4 TR mice) as previously described (Hoe et al., 2009).

Statistical analyses. Scholl analysis and spine density data were analyzed with GraphPad prism 4 software using one-way ANOVA followed by a Bonferroni post hoc test. For spine length data, the KolmogorovSmirnov statistical test was used. Descriptive statistics were displayed as an expressed mean \pm SEM, with significance at $p<0.05$.

\section{Results}

ApoE4 decreases while apoE3 increases spine density in primary hippocampal neurons

ApoE is involved in synapse and spine formation in primary cortical neurons (Brodbeck et al., 2008). To measure the effects of apoE isoforms, we transfected primary hippocampal neurons with GFP, treated them with purified apoE3, apoE4, or PBS for $48 \mathrm{~h}$, and then analyzed spine density. We found that apoE4 decreased spine density $(31 \pm 17.1 \%)$ and apoE3 increased spine density $(82 \pm 18.3 \%)$ compared with the control (supplemental Fig. $1 A, B$, available at www.jneurosci.org as supplemental mate- rial), consistent with a recent study (Brodbeck et al., 2008). We further measured the length of spines from the base of the neck to the furthest point on the spine head (Pak et al., 2001). The cumulative distribution of spine length showed a significant shift in spine distribution toward shorter spines for apoE4-treated neurons compared with control and apoE3 treatment (supplemental Fig. $1 C$, available at www.jneurosci.org as supplemental material). These findings suggest that apoE may play a critical role, not only in determining the appropriate number of spines, but also in spine morphogenesis, and thus synaptic plasticity.

\section{ApoE4 decreases spine density in vivo by 4 weeks of age}

Previous studies have uncovered an association between the apoE4 genotype and spine deficits in vivo (Ji et al., 2003; Lanz et al., 2003; Wang et al., 2005). However, these studies did not compare all three apoE isoforms or examine their effects on dendritic spines in the cortex. To examine this, we initially focused on 4-week-old APOE TR mice, analyzing pyramidal neurons in cortical layers II/III using the rapid Golgi impregnation method. Because spine density may vary within both the dendritic field of a single neuron and between different neurons (Alpár et al., 2006), the spines of the pyramidal neurons were counted from apical oblique (AO) and basal shaft (BS) dendrites (Fig. 1A,B). The densities of AO, BS, and total $(\mathrm{AO}+\mathrm{BS})$ dendritic spines 
4 Wks Hippocampus

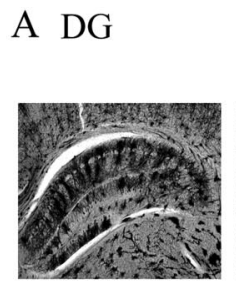

X2.5

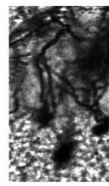

$\mathrm{X} 20$

$\mathrm{D}$

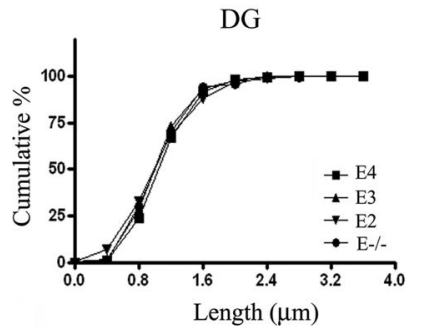

B

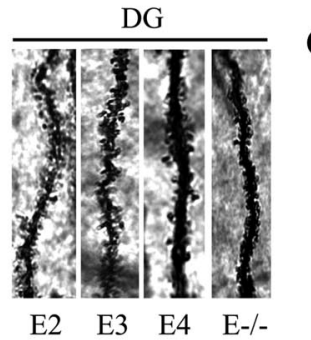

E CA1 BS

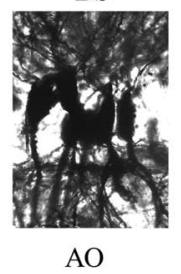

$\mathrm{AO}$
$\mathrm{F}$

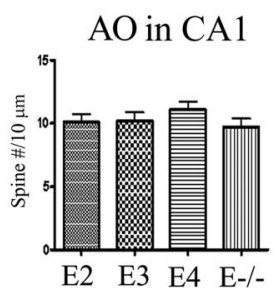

$\mathrm{C}$

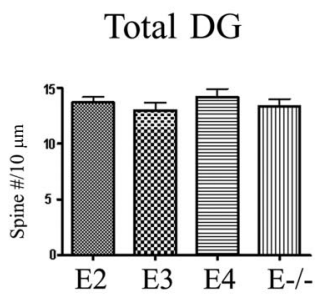

G

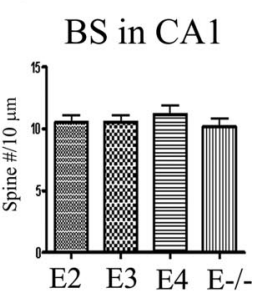

Figure 2. APOE isoforms exhibit similar spine density in the hippocampus at 4 weeks of age. Mouse brains were Golgi stained and granule cells in the dentate gyrus and CA1 neurons were imaged ( $n=4$ mice/genotype). $A$, Left, A Golgi impregnation of the hippocampus of an APOE3 TR mouse at $2.5 \times$. Right, A representative image of APOE3 TR mouse granule cells in the dentate gyrus at $20 \times$ magnification. $\boldsymbol{B}$, Representative dendrites for the dentate gyrus per genotype. $\boldsymbol{C}$, Averaged total spine density in the dentate gyrus (28 neurons/genotype). $\boldsymbol{D}$, The cumulative percentage distribution plots of spine length. $\boldsymbol{E}$, Representative image of AP0E3 TR CA1 neurons at $10 \times$ magnification. $\boldsymbol{F}$, Averaged data include quantification of A0 (28 neurons/genotype). G, BS (28 neurons/genotype) spine number per $10 \mu \mathrm{m}$ length.

were significantly decreased in APOE4 TR mice by $24-30 \%$ compared with APOE3 and APOE2 TR mice (Fig. $1 C-E$ ). APOEdeficient mice also had decreased $\mathrm{AO}, \mathrm{BS}$, and total spine densities by $28-33 \%$ compared with APOE3 and APOE2 TR mice (Fig. 1C-E). These data show that APOE4 TR mice had reduced cortical dendritic spine density similar to mice lacking apoE at young age.

Next, we examined the spine length to compare the morphology of individual dendritic spines in all three APOE TR mice and APOE-deficient mice. The cumulative distribution of spine length revealed a significant shift of APOE2 TR mice to longer spines compared with the other APOE TR and APOE-deficient mice (Fig. $1 F-H$ ).

To test whether apoE isoforms differentially affected spine formation in the hippocampus, we analyzed spine density and morphology in granule cells of the dentate gyrus of the hippocampus (Fig. $2 A, B$ ). The three APOE TR and the APOEdeficient mice did not alter the spine density in the dentate gyrus (Fig. 2C), nor were there significant morphological shifts in the distribution of spine length (Fig. 2D). We also examined the pyramidal cells in the CA1 region of the hippocampus (Fig. $2 E-$ $G)$. Again, all three APOE TR and APOE-deficient mice showed similar spine densities for CA1 neurons, suggesting that the impact of APOE on spine formation may vary across brain regions.

\section{ApoE4 further reduces spine density in the cortex at 1 year}

To examine whether the effect of apoE isoforms on spine formation was age dependent, we measured the spine density from all three APOE TR mice at 3 months and 1 year. Consistent with our observations at 4 weeks, APOE4 TR mice had decreased spine density at 3 months and a trend toward an even greater decrease at 1 year compared with APOE3 TR mice (Fig. $3 A, E)(24.4 \pm$ $8.8 \%$ and $55.6 \pm 10.5 \%$, respectively). APOE2 TR mice showed similar spine densities to APOE3 TR mice at both ages (Fig. 3A-
$C, E-G)$. For the spine morphology, we found that there are no significant differences in spine length distribution across groups at 3 months (Fig. 3D). However, APOE4 TR mice had significantly shorter spines compared with the other APOE TR mice at 1 year of age (Fig. $3 H$ ), consistent with our in vitro findings (supplemental Fig. 1, available at www.jneurosci.org as supplemental material). Overall, these data suggest that the effect of apoE4 on spine formation may increase with aging in the cortex.

We also tested whether apoE4 affected the spine density and morphology in the hippocampus with aging. We observed that all three APOE TR mice had similar spine densities at 3 months and 1 year for the dentate gyrus (Fig. $3 I, K$ ). We also found that there was no significant shift in distribution for spine length from all three APOE TR mice at 3 months (Fig. 3J). However, APOE2 TR mice had a significant shift in distribution to shorter spines in the dentate gyrus compared with APOE3 and APOE4 TR mice at 1 year (Fig. $3 L)$.

In summary, a comparison in cortical spine density for all three APOE TR mice over time (supplemental Fig. $2 \mathrm{~A}$, available at www.jneurosci.org as supplemental material) shows that by 4 weeks of age, APOE4 TR mice have a $27.7 \pm 7.4 \%$ decrease in spine density compared with APOE3 TR mice. By 1 year, ApoE4 TR mice had a further decrease in spine density by $55.6 \pm 10.5 \%$ compared with APOE3 TR mice (supplemental Fig. $2 A$, available at www.jneurosci.org as supplemental material). This suggests an increasing trend of APOE4 effects on pyramidal spines in cortical layer II/III with aging. However, all three APOE TR mice had similar spine densities over time in the dentate gyrus (supplemental Fig. $2 B$, available at www.jneurosci.org as supplemental material).

\section{ApoE4 decreases dendritic arborization in cortical neurons at 4 weeks}

ApoE3 increases dendritic arborization, while apoE4 decreases dendritic arborization in vitro (Nathan et al., 1994). We tested whether similar results were found in vivo. We used the Neurolucida software to trace dendrites of Golgi-stained pyramidal neurons in layers II/III of the somatosensory cortex at 4 weeks of age (Fig. 4A). Quantitative analysis revealed that apoE isoforms significantly affected elaboration of apical dendrites but not basal dendrites of these neurons. Apical branching was significantly increased in APOE2 TR mice compared with APOE3 (25.5 \pm $14.5 \%)$ and APOE4 (39.8 $\pm 11.7 \%)$ TR mice (Fig. 4 B). Furthermore, total apical dendritic length was significantly decreased in APOE4 compared with APOE2 TR mice (Fig. 4C). Scholl analysis did not reveal a difference in the complexity of apical dendrites between APOE3 and APOE4 TR mice. However, we observed that APOE3 TR mice had significantly decreased apical branching at proximal distances from the soma, whereas APOE4 TR mice had significantly decreased apical branching distally from the soma (Fig. 4D). 


\section{3 mths Cortical Layers II/III}

A

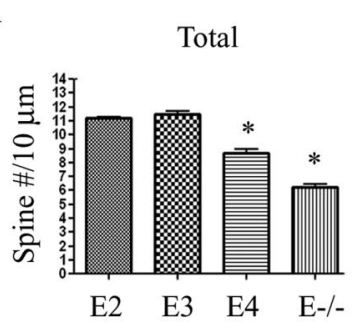

B

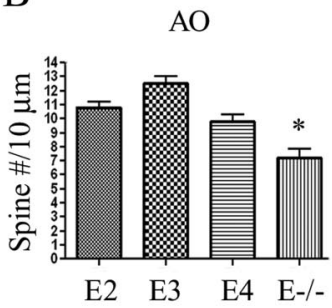

C

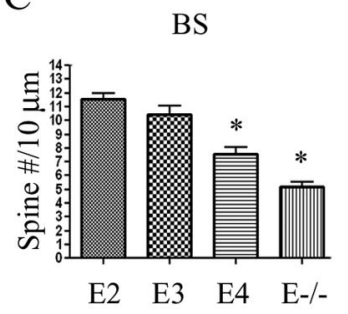

D

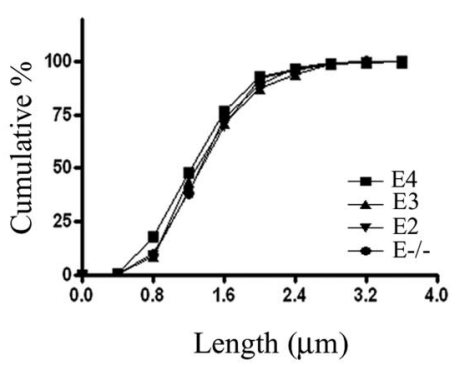

1 yr Cortical Layers II/III

E

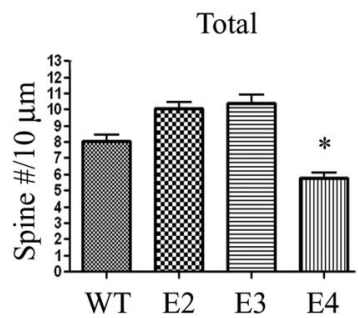

F

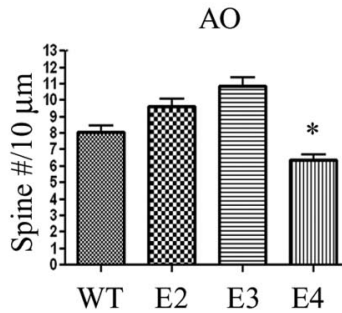

G

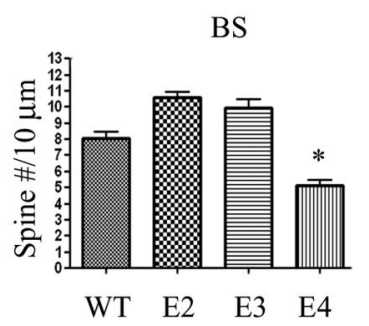

$\mathrm{H}$

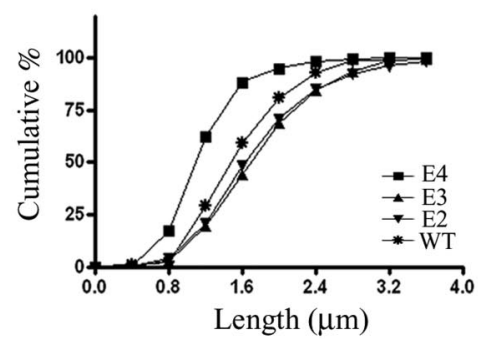

\section{3 mths Hippocampus}

I

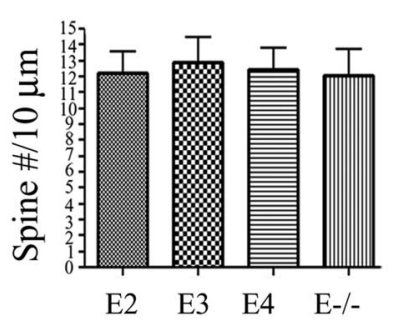

\section{J}

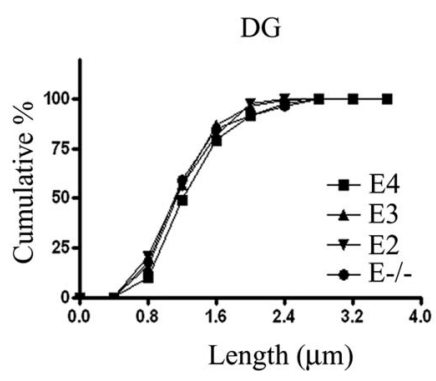

1 yr Hippocampus

$\mathrm{K}$

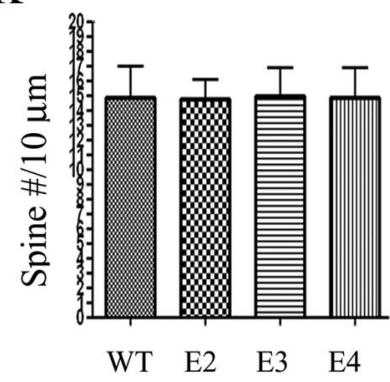

$\mathrm{L}$

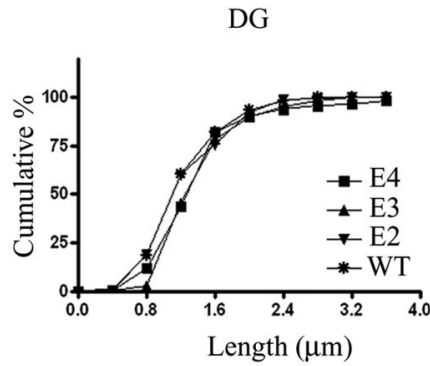

Figure 3. ApoE4 further reduces spine density in the cortex at 1 year. $A$, Averaged total spine density ( 56 neurons/group). B, Averaged A0 spine density (28 neurons/group). $C$, Averaged BS spine density (28 neurons/group). ${ }^{*} p<0.05$ compared with APOE3. D, Cumulative distribution of spine length. $\boldsymbol{E}-\mathbf{G}$, Mouse brains were Golgi stained and cortical layers $I / / I l l$ imaged at 1 year of age $(n=$ 4 mice/genotype). $\boldsymbol{E}$, Averaged data include total spine density ( 56 neurons/genotype). $\boldsymbol{F}$, Averaged data include A0 spine density (28 neurons/genotype). $\boldsymbol{G}$, Averaged data include BS spine density (28 neurons/genotype). ${ }^{*} p<0.05$ compared with APOE3. $\boldsymbol{H}$, Cumulative percentage distribution of spine length. APOE4 TR mice had a significant shift in distribution toward smaller spines compared with the other APOE TR (E3, E2) mice at 1 year of age. $I, J$, Mouse brains were Golgi stained and granule cells in the dentate gyrus were imaged at 3 months $(n=4) . I$, Averaged total spine density for all genotypes (28 neurons/group). J, Cumulative percentage distribution of spine length. $\boldsymbol{K}, \boldsymbol{L}$, Mouse brains were Golgi stained and granule cells in the dentate gyrus were imaged at 1 year $(n=4) . \boldsymbol{K}$, Averaged total spine density (28 neurons/group). $\boldsymbol{L}$, Cumulative percentage distribution for spine length. APOE2 TR had a significant shift in distribution toward shorter spines compared with APOE (E3, E4) TR mice.

\section{Discussion}

In the present study, we defined a physiological effect of apoE isoforms on spine formation in vivo. We demonstrate that APOE4 TR mice have reduced spine density and dendritic arborization in layer II/III of the cortex compared with APOE2 and APOE3 TR mice. We observed this deficit in three independent cohorts of mice at different ages ( 4 weeks, 3 months, and 1 year). These data suggest that apoE isoforms differentially affect spine formation and maintenance.

The effect of apoE isoforms on spine formation in vitro and in vivo

Several studies have suggested that apoE may play an important role in promoting synapse formation and synaptic plasticity (Nathan et al., 1994; Teter et al., 1999). Indeed, we and others have found that apoE4 decreased spine density compared with apoE3 in primary neurons (supplemental Fig. 1, available at www.jneurosci.org as supplemental material) (Brodbeck et al., 2008). Additionally, we and others have examined the effect of apoE4 on spine formation in vivo (Ji et al., 2003; Wang et al., 2005). We found that APOE4 TR mice have decreased spine density compared with APOE2 and APOE3 TR mice in the cortex at three different ages (Figs. 1, 3). APOE4 TR mice also have significantly reduced spine density compared with APOE3 TR mice in the amygdala at 7 months of age (Wang et al., 2005). Transgenic hAPP mice crossed with hAPOE2-overexpressing mice exhibit increased dendritic spine density in CA1 neurons (Lanz et al., 2003). Furthermore, APOE4 transgenic mice (under control of the GFAP promoter) have reduced spine density at 12 months and 24 months but not at 3 weeks compared with APOE3 trans- 


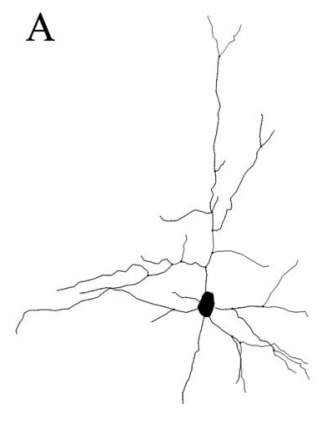

ApoE2

$\mathrm{B}$

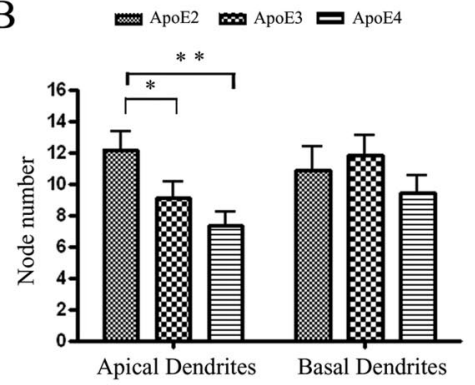

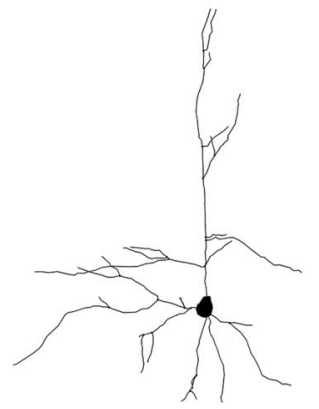

ApoE3

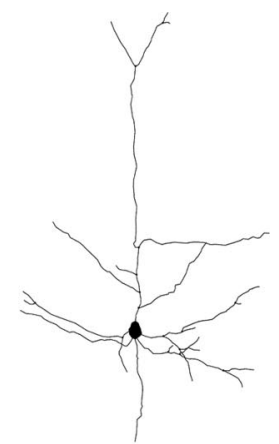

ApoE4

\section{$\mathrm{C}$}

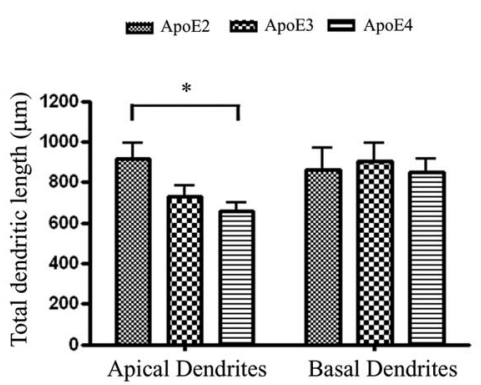

$\mathrm{D}$

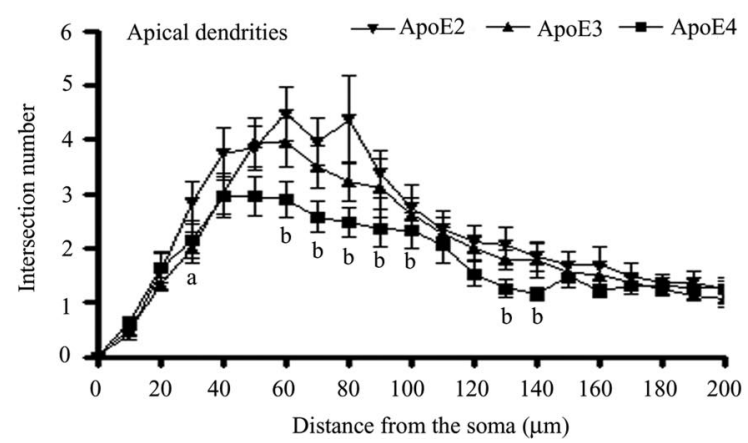

Figure 4. ApoE4 decreases dendritic arborization in cortical neurons at 4 weeks. A, Three-dimensional graphical tracing representing dendrite morphology. The number of apical and basal dendrites was measured in micrometers, and the number of apical and basal nodes was counted. $\boldsymbol{B}$, Averaged data include the node (branch) number for apical and basal dendrites for the three genotypes, analyzing 18 neurons per group. APOE2 mice had increased branching compared with APOE3 $(25.5 \pm 14.5 \%)$ and APOE4 (39.8 $\pm 11.7 \%)$. C, Averaged total dendritic length for apical and basal dendrites for the three genotypes, analyzing 18 neurons per group. APOE2 mice had significantly increased length ( $29.6 \pm 9.6)$ compared with APOE4 TR mice. ${ }^{*} p<0.05$, ${ }^{* *} p<0.01$. D, Using Scholl analysis, we graphed the average intersections per shell per neuron against the distance from the soma (in micrometers). ${ }^{a} p<0.05$ for APOE3 versus APOE2. ${ }^{b} p<0.05$ for APOE4 versus APOE2.

genic mice in the dentate gyrus (Ji et al., 2003). We found no difference in spine density across all three APOE genotypes in the dentate gyrus, at all ages, or in the CA1 region at 4 weeks (Figs. 2, $3 I-L)$. This discrepancy may be partially due to varying apoE levels found in the animal models used. For instance, we used APOE4 TR mice, which exhibit reduced brain apoE levels compared with APOE2 TR and APOE3 TR mice (Riddell et al., 2008), while Ji et al. (2003) used GFAP-APOE3 and GFAP-APOE4 mice, which express equal levels of apoE. Additionally, apoE protein levels are higher in the hippocampus compared with cortex regardless of APOE genotype for the APOE TR mice (Riddell et al., 2008; Sullivan et al., 2009). The decreased baseline apoE levels in the cortex compared with the hippocampus for APOE4 TR mice may account for the differential effects we observe in these brain regions. It is also possible that there is a discrepancy in expression levels of apoE receptors, which are implicated in synaptic plasticity, in the hippocampus versus the cortex (Qiu et al., 2006). Finally, we cannot completely exclude the possibility that the

discrepancy may be due to technical issues. Since neurons in hippocampus are more densely packed than those in cortex, imaged dendrites were more distal from the soma than those imaged in cortex.

We are the first to analyze spine morphology by examining spine length across all APOE genotypes in vivo. We found a variation in cortical layers II/III across genotypes at three different time points. Interestingly, APOE2 TR mice had a significant distribution shift toward longer spines in the cortex at 4 weeks of age compared with the other APOE TR mice. This difference was not evident at later time points, suggesting a role for apoE2 in the early development of spines. Conversely, APOE4 TR mice had a significant distribution shift toward shorter spines compared with APOE3 and APOE2 TR mice at 1 year. This was not observed at earlier time points, suggesting that apoE4's detrimental effects on spine morphology and spine density in the cortex may build over time.

How do apoE isoforms differentially regulate spine density? ApoE binds to a family of low-density lipoprotein receptors, which are involved in neurite outgrowth and long-term potentiation (Qiu et al., 2006). A recent study found differences in intracellular signaling cascades in all three APOE TR mice, suggesting that this may result from their differential binding affinities to this family of receptors (Korwek et al., 2009). Another explanation could be that the apoE4 is deficient in providing neurons with high-density lipid particles, which are necessary for dendritic spine remodeling and the formation of mature synapses. (Mauch et al., 2001).

\section{The effect of apoE isoforms on neurite development}

In previous studies, apoE4 decreased dendritic length and arborization compared with apoE3 in vitro (Nathan et al., 1994; Teter et al., 1999). However, we did not observe a significant difference in dendritic complexity between APOE3 and APOE4 TR mice, although some difference was noted. This may be due to several factors such as age and brain region examined. For example, we compared neurite arborization in APOE TR mice in the somatosensory cortical layer II/III at 4 weeks compared with the amygdala at 7 months in a previous study (Wang et al., 2005). Interestingly, we observed that APOE3 and APOE4 TR mice have decreased apical branching compared with APOE2 TR mice (Fig. 4). Moreover, APOE4 TR mice have decreased apical dendritic length compared with APOE2 TR mice (Fig. 4). However, we did not observe a significant difference in basal dendrites across all genotypes. Similarly, there were alterations in apical but not basal dendritic morphology compared with controls in a transgenic $\mathrm{AD}$ mouse model (Alpár et al., 2006). This could be related to the fact that basal dendrites have less complexity than apical dendrites (Henze et al., 1996). 


\section{Conclusion}

In conclusion, this study is the first to demonstrate that apoE isoforms have a differential regulatory effect on dendritic complexity and spine formation in cortical neurons. APOE4 TR mice have reduced apical arborization compared with APOE2 TR mice. Additionally, APOE4 TR mice have reduced spine density compared with APOE2 and APOE3 TR mice. This reduction in spine density for APOE4 TR mice increases over time, with significantly smaller spines than the other APOE TR mice at 1 year. These data may inform both normal brain functions of apoE and differential reparative responses, dependent on ApoE isoform, to acute and chronic brain injuries, including AD.

\section{References}

Alpár A, Ueberham U, Brückner MK, Seeger G, Arendt T, Gärtner U (2006) Different dendrite and dendritic spine alterations in basal and apical arbors in mutant human amyloid precursor protein transgenic mice. Brain Res 1099:189-198.

Bellosta S, Nathan BP, Orth M, Dong LM, Mahley RW, Pitas RE (1995) Stable expression and secretion of apolipoproteins E3 and E4 in mouse neuroblastoma cells produces differential effects on neurite outgrowth. J Biol Chem 270:27063-27071.

Brodbeck J, Balestra ME, Saunders AM, Roses AD, Mahley RW, Huang Y (2008) Rosiglitazone increases dendritic spine density and rescues spine loss caused by apolipoprotein E4 in primary cortical neurons. Proc Natl Acad Sci U S A 105:1343-1346.

Buttini M, Orth M, Bellosta S, Akeefe H, Pitas RE, Wyss-Coray T, Mucke L, Mahley RW (1999) Expression of human apolipoprotein E3 or E4 in the brains of Apoe-/- mice: isoform-specific effects on neurodegeneration. J Neurosci 19:4867-4880.

Daniele A, Matera MG, Seripa D, Acciarri A, Bizzarro A, Pilotto A, Masullo C (2009) APOE $\varepsilon 2 / \varepsilon 4$ genotype a risk factor for primary progressive aphasia in women. Arch Neurol 66:910-912.

DeKosky ST, Scheff SW (1990) Synapse loss in frontal cortex biopsies in Alzheimer's disease: correlation with cognitive severity. Ann Neurol 27: 457-464.

Filippini N, MacIntosh BJ, Hough MG, Goodwin GM, Frisoni GB, Smith SM, Matthews PM, Beckmann CF, Mackay CE (2009) Distinct patterns of brain activity in young carriers of the APOE-epsilon4 allele. Proc Natl Acad Sci U S A 106:7209-7214.

Henze DA, Cameron WE, Barrionuevo G (1996) Dendritic morphology and its effects on the amplitude and rise-time of synaptic signals in hippocampal CA3 pyramidal cells. J Comp Neurol 369:331-344.

Herz J, Beffert U (2000) Apolipoprotein E receptors: linking brain development and Alzheimer's disease. Nat Rev Neurosci 1:51-58.

Hoe HS, Lee KJ, Carney RS, Lee J, Markova A, Lee JY, Howell BW, Hyman BT, Pak DT, Bu G, Rebeck GW (2009) Interaction of reelin with amyloid precursor protein promotes neurite outgrowth. J Neurosci 29:74597473.

Ji Y, Gong Y, Gan W, Beach T, Holtzman DM, Wisniewski T (2003) Apolipoprotein E isoform-specific regulation of dendritic spine morphology in apolipoprotein E transgenic mice and Alzheimer's disease patients. Neuroscience 122:305-315.

Korwek KM, Trotter JH, LaDu MJ, Sullivan PM, Weeber EJ (2009) ApoE isoform-dependent changes in hippocampal synaptic function. Mol Neurodegener 4:21.

Lanz TA, Carter DB, Merchant KM (2003) Dendritic spine loss in the hip- pocampus of young PDAPP and Tg2576 mice and its prevention by the ApoE2 genotype. Neurobiol Dis 13:246-253.

Mauch DH, Nägler K, Schumacher S, Göritz C, Müller EC, Otto A, Pfrieger FW (2001) CNS synaptogenesis promoted by glia-derived cholesterol. Science 294:1354-1357.

Nathan BP, Bellosta S, Sanan DA, Weisgraber KH, Mahley RW, Pitas RE (1994) Differential effects of apolipoproteins E3 and E4 on neuronal growth in vitro. Science 264:850-852.

Pak DT, Yang S, Rudolph-Correia S, Kim E, Sheng M (2001) Regulation of dendritic spine morphology by SPAR, a PSD-95-associated RapGAP. Neuron 31:289-303.

Plassman BL, Welsh-Bohmer KA, Bigler ED, Johnson SC, Anderson CV, Helms MJ, Saunders AM, Breitner JC (1997) Apolipoprotein E epsilon 4 allele and hippocampal volume in twins with normal cognition. Neurology 48:985-989.

Poirier J, Davignon J, Bouthillier D, Kogan S, Bertrand P, Gauthier S (1993) Apolipoprotein E polymorphism and Alzheimer's disease. Lancet 342: 697-699.

Qiu S, Korwek KM, Weeber EJ (2006) A fresh look at an ancient receptor family: emerging roles for low density lipoprotein receptors in synaptic plasticity and memory formation. Neurobiol Learn Mem 85:16-29.

Rebeck GW, Reiter JS, Strickland DK, Hyman BT (1993) Apolipoprotein E in sporadic Alzheimer's disease: allelic variation and receptor interactions. Neuron 11:575-580.

Riddell DR, Zhou H, Atchison K, Warwick HK, Atkinson PJ, Jefferson J, Xu L, Aschmies S, Kirksey Y, Hu Y, Wagner E, Parratt A, Xu J, Li Z, Zaleska MM, Jacobsen JS, Pangalos MN, Reinhart PH (2008) Impact of apolipoprotein E (ApoE) polymorphism on brain ApoE levels. J Neurosci 28:11445-11453.

Scarmeas N, Habeck CG, Hilton J, Anderson KE, Flynn J, Park A, Stern Y (2005) APOE related alterations in cerebral activation even at college age. J Neurol Neurosurg Psychiatry 76:1440-1444.

Strittmatter WJ, Saunders AM, Schmechel D, Pericak-Vance M, Enghild J, Salvesen GS, Roses AD (1993) Apolipoprotein E: high-avidity binding to beta-amyloid and increased frequency of type 4 allele in late-onset familial Alzheimer disease. Proc Natl Acad Sci U S A 90:1977-1981.

Sullivan PM, Mezdour H, Aratani Y, Knouff C, Najib J, Reddick RL, Quarfordt SH, Maeda N (1997) Targeted replacement of the mouse apolipoprotein E gene with the common human APOE3 allele enhances diet-induced hypercholesterolemia and atherosclerosis. J Biol Chem 272: 17972-17980.

Sullivan PM, Han B, Liu F, Mace BE, Ervin JF, Wu S, Koger D, Paul S, Bales KR (2009) Reduced levels of human apoE4 protein in an animal model of cognitive impairment. Neurobiol Aging. Advance online publication. Retrieved November 18, 2009. doi:10.1016/j.neurobiolaging.2009.05.011.

Teasdale GM, Nicoll JA, Murray G, Fiddes M (1997) Association of apolipoprotein E polymorphism with outcome after head injury. Lancet 350: $1069-1071$.

Teter B, Xu PT, Gilbert JR, Roses AD, Galasko D, Cole GM (1999) Human apolipoprotein $\mathrm{E}$ isoform-specific differences in neuronal sprouting in organotypic hippocampal culture. J Neurochem 73:2613-2616.

Wang C, Wilson WA, Moore SD, Mace BE, Maeda N, Schmechel DE, Sullivan PM (2005) Human apoE4-targeted replacement mice display synaptic deficits in the absence of neuropathology. Neurobiol Dis 18:390-398.

Yoshizawa T, Yamakawa-Kobayashi K, Komatsuzaki Y, Arinami T, Oguni E, Mizusawa H, Shoji S, Hamaguchi H (1994) Dose-dependent association of apolipoprotein E allele epsilon 4 with late-onset, sporadic Alzheimer's disease. Ann Neurol 36:656-659. 